
the Non-Dominant Eye on the Binocular Defocus Curve with a Non-Diffractive Extended Vision Intraocular Lens

\author{
Kjell G Gundersen (D) \\ Richard Potvin (D) ${ }^{2}$ \\ 'IFocus Øyeklinikk AS, Haugesund, \\ Norway; ${ }^{2}$ Science in Vision, Bend, \\ OR, USA
}

Correspondence: Kjell G Gundersen Emailkg@ifocus.no
Purpose: To quantify the changes in the binocular defocus curve associated with the
Vivity ${ }^{\mathrm{TM}}$ non-diffractive extended vision intraocular lens when the dominant eye was
targeted for emmetropia and the non-dominant eye was artificially targeted for slight myopia
using spectacles.
Patients and Methods: This was a non-interventional research study of the corrected binocular defocus curve associated with binocular emmetropia (Setting A) and with emmetropia in the dominant eye and two different levels of myopia simulated in the non-dominant eye ( -0.50 D, Setting B and -1.00 D, Setting C). Subjects were patients implanted with the AcrySof $^{(B)}$ IQ Vivity ${ }^{(B)}$ intraocular lens in both eyes 3 to 12 months previously. Using the defocus data, the percentage of subjects with a continuous $2.5 \mathrm{D}$ range of vision (distance to $40 \mathrm{~cm}$ ) was calculated for various levels of minimum visual acuity (VA).

Results: Forty subjects were enrolled. The mean spherical equivalent refraction was -0.06 $\mathrm{D} \pm 0.36 \mathrm{D}$, with $0.37 \mathrm{D} \pm 0.29 \mathrm{D}$ of refractive cylinder. There was no statistically significant difference in the mean VA at $-0.25 \mathrm{D}$ or at $-0.50 \mathrm{D}$ vergences between the test Settings, but there was a statistically significant difference at all other vergences. Differences were particularly noticeable at $-2.00 \mathrm{D},-2.50 \mathrm{D}$ and $-3.00 \mathrm{D}$, where higher myopia in the nondominant eye yielded better binocular VA. A $2.5 \mathrm{D}$ range of functional vision (20/25) was achieved by $38 \%$ of subjects at Setting A, $68 \%$ of subjects at Setting B and $85 \%$ of subjects at Setting C. At setting C, all but one subject (39/40, 97.5\%) had a $2.5 \mathrm{D}$ range of vision with a VA of $20 / 32$ or better.

Conclusion: Significant gains in binocular near vision, with only a nominal effect on distance vision, can be achieved with the Vivity IOL by leaving the non-dominant eye of patients with $0.50 \mathrm{D}$ or $1.00 \mathrm{D}$ of myopia.

Keywords: non-diffractive, extended vision, extended depth of focus, presbyopia, cataract

\section{Plain Language Summary}

At the time of cataract surgery many patients would like to reduce their dependence on glasses for distance, intermediate (computer) or near (reading) work. There are several different options for patients to consider, including lenses that extend the distance focus so that computer work is easier, though near vision may not be sufficient for work without glasses. By making one eye a bit short-sighted, binocular (both eyes) near vision can be improved. The current study was designed to compare measured vision at distances from far to near when the eyes were both corrected for good distance vision, and when one eye was made short-sighted, simulated using glasses. 
Results showed that making one eye a little short sighted with glasses did improve the near vision, as expected, with only a small change in the binocular distance vision. Having one eye corrected to be slightly short-sighted may be of interest to patients who want greater spectacle freedom when this lens is implanted.

\section{Introduction}

Many patients presenting for cataract surgery are interested in reducing or eliminating their need for spectacles after surgery. There are numerous options for them to consider, from bifocal or trifocal intraocular lenses, to extended depth of focus (EDOF) intraocular lenses, to a monovision strategy using monofocal intraocular lenses - correcting the non-dominant eye so that it is slightly myopic. Some surgeons will use a combination of these approaches. ${ }^{1,2}$ The multifocal IOLs, particularly the trifocals, generally provide the highest likelihood of complete spectacle independence. ${ }^{3-5}$ EDOF lenses are likely to provide good vision at distance and intermediate, with slightly worse near vision, but the expectation is that visual disturbances will be lower with an EDOF IOL because there are not two (or three) distinct foci. ${ }^{5}$ This expectation may be in error. Several studies of the Symfony $^{\circledR}$ EDOF lens (Johnson \& Johnson Vision, Santa Ana, CA) have shown that the visual disturbances experienced by patients are not significantly different than for a trifocal IOL. ${ }^{6-8}$ This may be due to the diffractive nature of this EDOF design. Patients tend to have more visual disturbances with trifocal IOLs, and the Symfony EDOF IOL, than with monofocal IOLs. ${ }^{6,7}$ The limitation of reduced near vision with EDOF lenses can be mitigated by targeting one eye (usually the nondominant eye) for slight myopia, but this may introduce concerns such as an increase in visual disturbances reported. ${ }^{9}$

An alternative, non-diffractive, option for extending the depth of focus of an IOL is using a small aperture, such as is incorporated into the IC-8 IOL (Acufocus, Inc., Irvine, CA). ${ }^{10}$ This provides a similar effect to "stopping down" a camera. The IC-8 IOL achieves the objective of an extended depth of focus, but at the expense of limiting the light entering the eye. This may be problematic in dim light conditions - at present, this IOL is generally implanted only monocularly. Diffractive effects at the edge of the small aperture will also have some negative effect on overall visual quality.
Modifying the spherical aberration (SA) of an IOL can also produce an extended depth of focus effect. Several different designs incorporating this technology have been developed, including an "aberration free IOL" that counts on the naturally occurring corneal SA to produce an EDOF effect, ${ }^{11}$ and an IOL that alternates annular zones of positive and negative spherical aberration. ${ }^{12}$ There are limits to the range of focus that can be produced in this manner, as higher levels of SA will reduce the overall quality of vision and result in halos. In addition, corneal spherical aberration can vary greatly from eye to eye, so a consistent effect between eyes is problematic.

The AcrySof ${ }^{\circledR}$ IQ Vivity ${ }^{\mathrm{TM}}$ IOL utilizes an entirely different method to produce an extended depth of focus. Wavefront shaping technology is in common use in industry to alter the wavefront from a light source. For instance, a laser beam might have a Gaussian intensity profile (much like a bell curve in statistics) as it exits the laser. Wavefront shaping can be used further along the optical path to "square off" this profile, improving the laser's ability to etch more sharply, with less rounding at the edges. Alcon has applied this wavefront shaping concept to modify the optics of an intraocular lens (X-WAVE ${ }^{\mathrm{TM}}$ technology) to produce an extended focal length that also focuses almost all the light from the IOL in that specific range. This reduces the potential for visual disturbances because visual disturbances such as halos are generally a function of the "unused" light that is not in focus. ${ }^{13}$ Visual disturbances with this non-diffractive extended vision (NDEV) IOL have been shown to be not statistically significantly different from a monofocal IOL. ${ }^{14}$ Intermediate vision was significantly better than can be achieved with a monofocal IOL. However, as with the Symfony IOL, the near VA was better than can be achieved with a monofocal IOL but worse than can be achieved with a trifocal or bifocal IOL. ${ }^{14}$

One well established method to improve near vision with any IOL is to make one eye slightly myopic (monovision); this would typically be applied for IOLs that provide insufficient near vision (eg, EDOF or monofocal IOLs). The binocular defocus curve for the Vivity IOL ${ }^{14}$ suggests that a myopic shift of $0.50 \mathrm{D}$ to $1.00 \mathrm{D}$ would be sufficient to provide a mean near visual acuity of 0.1 $\log$ MAR (20/25, 0.8 decimal). This appears consistent with the recommendations of Cochener et al with respect to the Symfony IOL. ${ }^{15}$ The other EDOF lenses mentioned above have also been clinically tested in a monovision modality. 
The purpose of the current study was to determine how artificially introducing low levels of myopia (with a spectacle lens) in the non-dominant eye of a patient bilaterally implanted with the Vivity IOL affects visual acuity at different vergences, and how the overall range of vision is affected. This is the first study of its kind with this lens.

\section{Patients and Methods}

This study was a single-arm, non-interventional study of visual outcomes after successful bilateral implantation of the Vivity non-diffractive extended vision (NDEV) IOL. Regional ethics committee approval for the study was obtained from the Regionale komiteer for medisinsk og helsefaglig forskningsetikk (REK) Norway, and all subjects signed an approved informed consent document. The study was non-interventional, so there was no requirement to register it as a clinical trial. The study was conducted in accordance with good clinical practice and followed the tenets of the Declaration of Helsinki. Data are not available for sharing.

Eligible patients had to have had previous uncomplicated surgery with normal outcomes, a best-corrected binocular and monocular distance visual acuity (VA) of 20/40 (0.3 logMAR) or better and have had surgery between 3 months and 12 months previously. Subjects with ocular pathology, previous corneal surgery, or significant posterior capsular opacity (PCO) were excluded. All subjects had surgery performed by one surgeon (KGG). Eyes with both toric and non-toric IOLs were included. Both eyes of a subject had to meet the inclusion/exclusion criteria.

Subjects were recruited from the site's clinical records from the previous year of surgery and, if they met the inclusion and exclusion criteria above, they were asked to participate in two postoperative diagnostic visits between one and three weeks apart (to reduce the potential effects of fatigue). Clinical evaluations included the manifest refraction, and both uncorrected and distance-corrected visual acuity at distance $(4 \mathrm{~m})$, intermediate $(66 \mathrm{~cm})$ and near $(50 \mathrm{~cm}$ and $40 \mathrm{~cm})$. Uncorrected low contrast visual acuity at distance was also tested in both photopic and mesopic conditions and patient reported outcomes were collected. The M\&S Technologies Clinical Trial Suite (Niles, IL, USA) was used for visual acuity data collection. Four binocular defocus curves were also collected for each subject: one with both eyes uncorrected, one with both eyes corrected for emmetropia, one with the non- dominant eye corrected to yield $-0.50 \mathrm{D}$ myopia and one with the non-dominant eye corrected to yield -1.00 D myopia. Eye dominance was determined using the Mile's test (sighting through a triangular opening formed by the hands extended to arm's length). Defocus curves were tested from $+1.00 \mathrm{D}$ to $-1.00 \mathrm{D}$ in $0.25 \mathrm{D}$ steps, and from $-1.00 \mathrm{D}$ to $-3.00 \mathrm{D}$ in $0.50 \mathrm{D}$ steps. Only the defocus data are reported here; the other clinical results will be reported elsewhere.

Relevant surgical planning data were extracted from the subjects' clinical records. In the majority of cases one eye was targeted using the least-plus surgical planning result and the other was targeted using the least-minus result. One optometrist conducted all testing during the diagnostic visits. Defocus curve data were collected and analyzed using an Access database (Microsoft Corp., Redmond, WA, USA) customized for the purpose. Detailed statistical analyses were performed using the STATISTICA data analysis software system, version 12 (TIBCO Software Inc., Palo Alto, CA, USA). An analysis of variance (ANOVA) was used to evaluate differences in VA at the various vergences tested in the defocus curve, with a significance level based on $p \leq 0.05$.

\section{Results}

A total 40 subjects were successfully recruited for the study. Table 1 contains a summary of the relevant demographic and refractive data. Scheduling issues resulted in several eyes being evaluated slightly later than one year postop (maximum 413 days), but this was not considered clinically important. There was no statistically significant difference in the postoperative mean refraction spherical equivalent (MRSE) or the postoperative refractive cylinder between the toric and

Table I Demographics, Refractive and VA Data ( $\mathrm{n}=40$ Subjects, 80 Eyes)

\begin{tabular}{|l|c|}
\hline Age (years) & $59 \pm 8(48$ to $8 \mathrm{I})$ \\
Male/Female & $25 / 15$ \\
Days post-surgery & $186 \pm 98(9 \mathrm{I}$ to $4 \mathrm{I} 3)$ \\
Non-toric/Toric & $68 / 12$ \\
MRSE (D) & $-0.06 \pm 0.36(-1.00$ to I.I2) \\
Cylinder (D) & $-0.37 \pm 0.29(-1.25$ to 0.00$)$ \\
Binocular UDVA & $-0.08 \pm 0.0 .07(-0.18$ to 0.12$)$ \\
Binocular CDVA & \\
Emmetropia & $-0.11 \pm 0.05(-0.20$ to 0.00$)$ \\
Non-dominant $-0.50 \mathrm{D}$ & $-0.07 \pm 0.06(-0.18$ to 0.04$)$ \\
Non-dominant $-1.00 \mathrm{D}$ & $-0.05 \pm 0.07(-0.20$ to 0.04$)$ \\
\hline
\end{tabular}

Abbreviations: VA, visual acuity; MRSE, mean refraction spherical equivalent; D, diopter; UDVA, uncorrected distance VA; CDVA, corrected distance VA. 


\section{Vertical bars denote 0.95 confidence intervals}

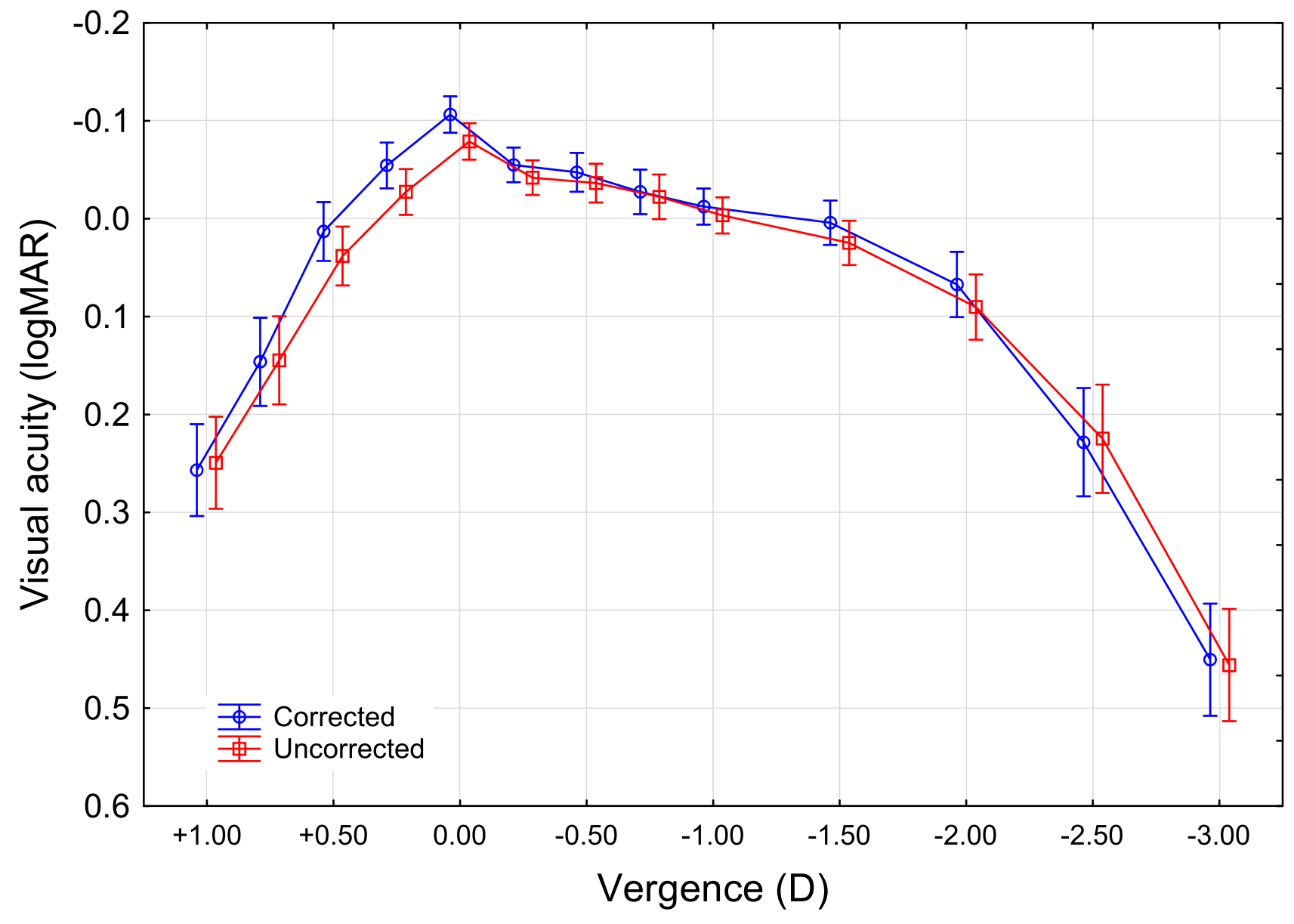

Figure I Defocus curves, binocular uncorrected and binocular best distance corrected.

non-toric eyes ( $p=0.51$ and 0.57 , respectively). There were no adverse events identified at any of the diagnostic visits.

Figure 1 shows the mean uncorrected binocular defocus curve along with the defocus curve when both eyes were corrected for emmetropia. As can be seen, the curves are very similar, with the only statistically significant difference being at Plano distance (vergence of 0.00 ), where the corrected VA was $0.03 \operatorname{logMAR}$, or about 1.5 letters, better than the uncorrected VA $(-0.11$ vs $-0.08, p=0.04)$. In both the corrected and uncorrected state, the binocular mean visual acuity was $0.1 \log$ MAR (20/25 Snellen, 0.8 decimal) or better through a vergence range from $0.0 \mathrm{D}$ to $-2.0 \mathrm{D}$, corresponding to a working range of distance to $50 \mathrm{~cm}$.

Figure 2 shows the binocular corrected defocus curves for three conditions: i) when the non-dominant eye was corrected for distance, ii) when the non-dominant eye was made $-0.50 \mathrm{D}$ myopic and iii) when the non-dominant eye was made $-1.00 \mathrm{D}$ myopic; in the latter two cases this was achieved with a trial frame. There was no statistically significant difference in the mean VA at $-0.25 \mathrm{D}$ or at $-0.50 \mathrm{D}$ between the test conditions, but there was a statistically significant difference at all other vergences (positive and negative). Differences were particularly noticeable at the $-2.00 \mathrm{D},-2.50 \mathrm{D}$ and $-3.00 \mathrm{D}$ vergences. As expected, the higher level of myopia in the non-dominant eye yielded much better binocular visual acuities at these vergences. Binocular corrected distance vision results (CDVA) for the three conditions, based on the zero vergence defocus result, are shown in Table 1. There was a statistically significant difference in the CDVA between the conditions $(p<0.01)$, but the maximum mean difference was 3 letters, or just over half a line of logMAR acuity. In all three conditions the mean binocular CDVA was better than $0.0 \log$ MAR $(20 / 20)$.

Using the measured VA data at various vergences, the range of vision provided (from distance to near) could be determined for each subject. For each level of myopia in the non-dominant eye (Emmetropia, $-0.50 \mathrm{D}$ or $-1.00 \mathrm{D}$ ) 


\section{Vertical bars denote 0.95 confidence intervals}

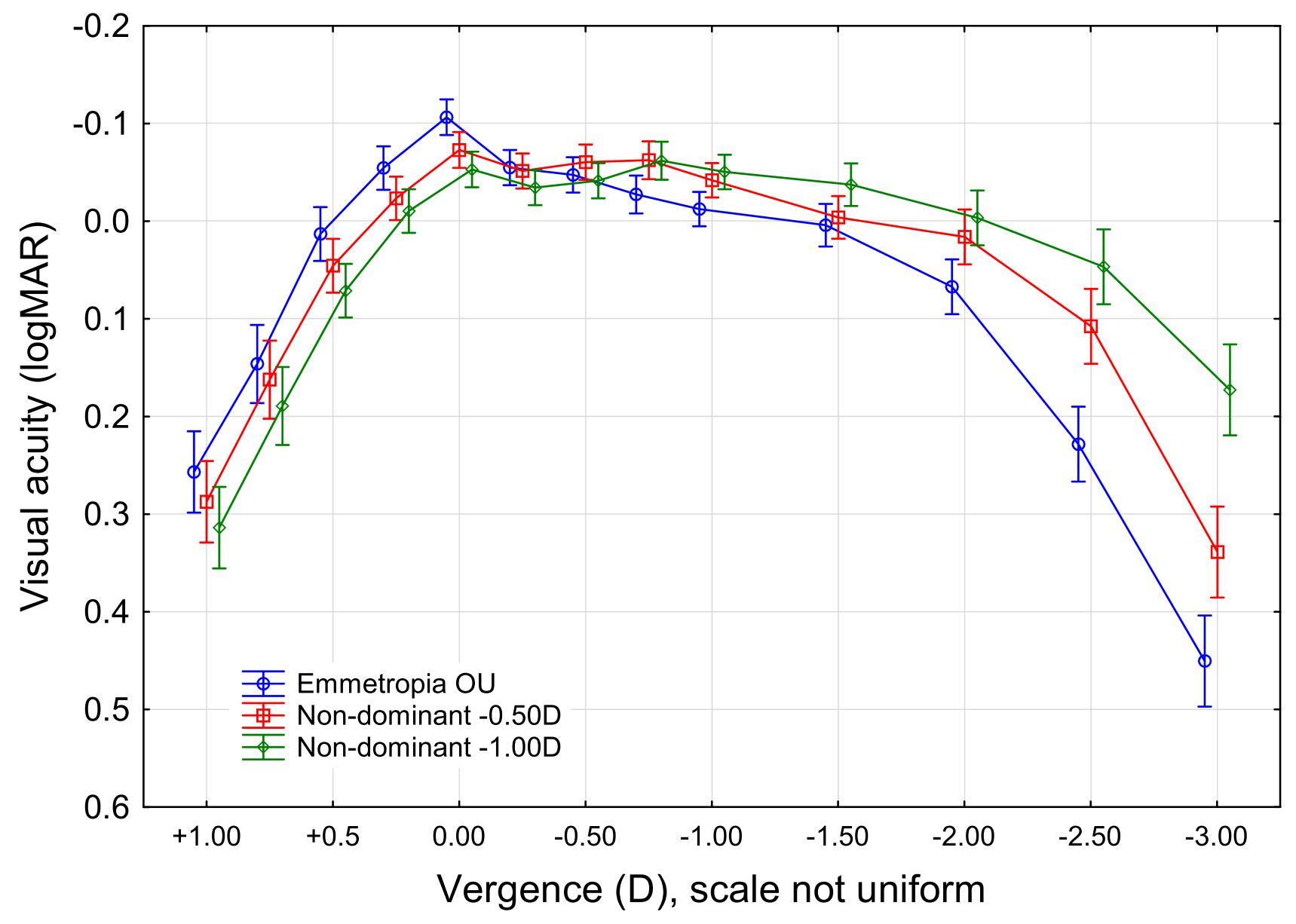

Figure 2 Defocus curves by correction of myopia in the non-dominant eye.

the percentage of subjects who had a binocular range of vision from $0.0 \mathrm{D}$ to $2.5 \mathrm{D}$ (a proxy for vision from distance to $40 \mathrm{~cm}$ ) was calculated for several different acuity limits. Results are shown in Figure 3. As can be seen, increasing the myopia in the non-dominant eye yielded significantly more eyes with a $2.5 \mathrm{D}$ range of vision at each tested level of acuity. For functional (0.1 $\log$ MAR, $20 / 25$ or 0.8 decimal) vision, $85 \%$ of subjects with a $-1.00 \mathrm{D}$ myopic correction in the non-dominant eye had a $2.5 \mathrm{D}$ range of vision, compared to only $38 \%$ of subjects when both eyes were corrected for emmetropia. All but one subject (39/40, 97.5\%) had visual acuity of 20/ 32 or better across that range of vergences.

\section{Discussion}

The results here indicate that the binocular defocus curve, and hence the functional range of vision, for this NDEV IOL can be significantly improved with only a modest myopic target in the non-dominant eye (maximum 1.0 D evaluated here), consistent with the findings of Cochener for a different EDOF lens. ${ }^{15}$ Achieving functional near vision with a minimal degree of ametropia ( $<1.50 \mathrm{D})$ between eyes helps to mitigate any potential negative impacts on stereo vision, contrast sensitivity or binocular rivalry, and reduces the potential for asthenopia if there is strong binocular rivalry or ocular dominance. ${ }^{16}$

Results with this lens and the levels of monovision tested appear better than were achieved with an aberration free lens based on spherical aberration that included a higher level of monovision in the non-dominant eye (mean $-1.38 \mathrm{D}) .^{11}$ The defocus curves with $-0.50 \mathrm{D}$ and $-1.00 \mathrm{D}$ in the non-dominant eye both also appear better than results for the IC-8 small aperture lens with 0.75 $\mathrm{D}$ of monovision ${ }^{17}$ and the Symfony lens with various levels of monovision. ${ }^{9,18}$ These results may be in part a function of the longer range of vision with the Vivity IOL measured here when both eyes were corrected for 


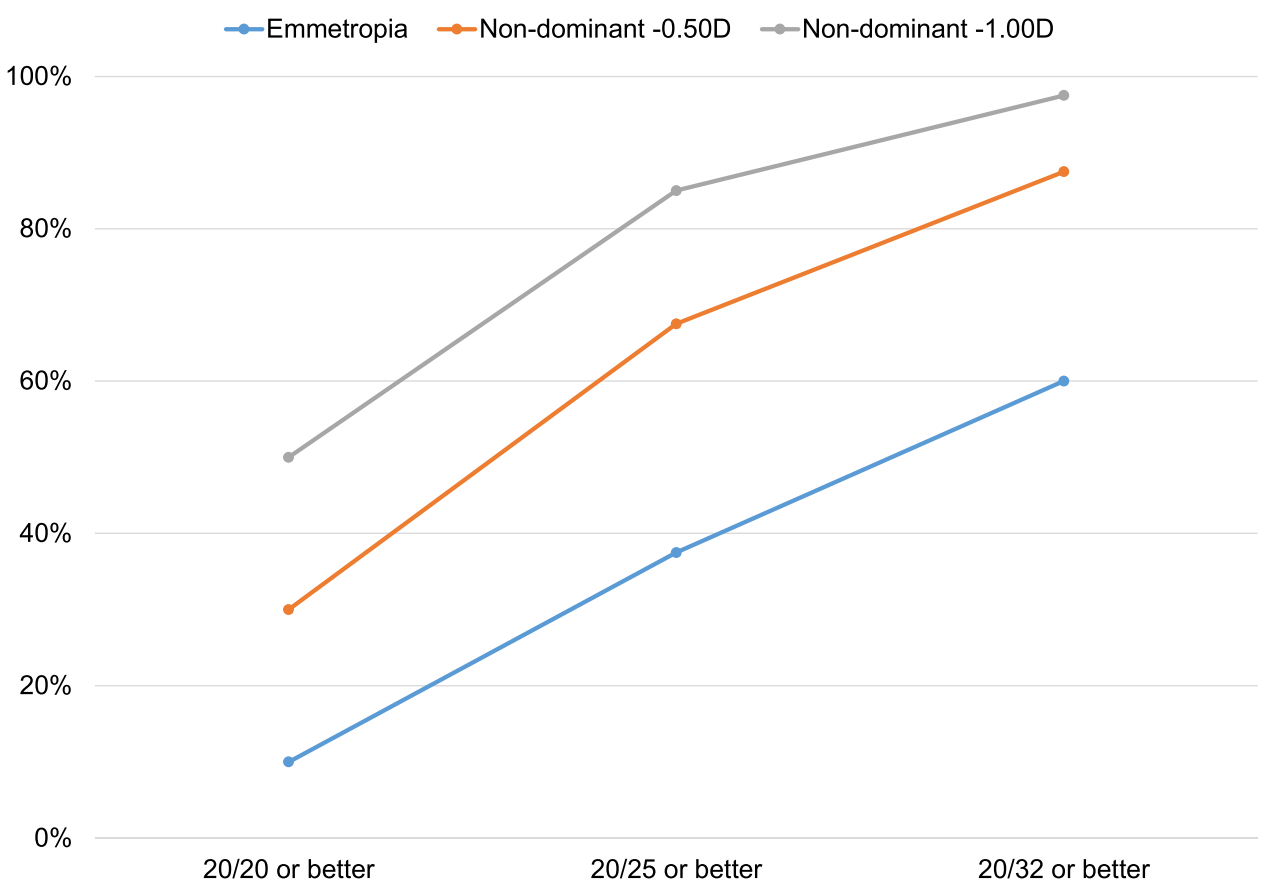

Figure 3 Percentage of eyes with a $2.5 \mathrm{D}$ range of vision $(0.0 \mathrm{D}$ to $-2.5 \mathrm{D})$ by visual acuity cutoff and correction of myopia in the non-dominant eye.

emmetropia. Results in the current study were about one line better at vergences of $-2.0 \mathrm{D}$ and $-2.50 \mathrm{D}$ than those reported in the Vivity Summary of Safety and Effectiveness document. ${ }^{14}$ Note that comparisons between studies should be interpreted with caution, as test conditions and measurement procedures can vary.

One of the challenges of this monovision approach, particularly when the levels of myopia are relatively low $(<1.0 \mathrm{D})$, is accurate biometry and IOL planning. A complicating factor appears to be that formulas may be slightly less reliable when targeting myopia instead of emmetropia. ${ }^{19}$ Targeting the first eye for emmetropia may be the best strategy, with the results from that eye influencing the target for the second eye. In this clinic surgeries are performed bilaterally at the same visit, so that would not be an option.

There are limitations to the current study. The charts were varied for the defocus curve testing the testing of each condition, but the testing order was not random. The myopia in the non-dominant eye was simulated, so the effects of the given levels of monovision on subjective quality of vision and visual disturbances could not be determined. In addition, the subjects' longer-term tolerance to the different levels of monovision could not be assessed. These two components can only be evaluated with subjects whose surgical planning is designed to leave them with a monovision result. This would be an important area for future research.

In conclusion, there appear to be significant gains in binocular near vision that can be achieved with this nondiffractive extended vision IOL by leaving the nondominant eye of patients with a modest level of monovision $(0.50 \mathrm{D}$ to $1.00 \mathrm{D})$. The relative effect on binocular distance vision appears nominal, with mean CDVA better than 20/20 and a relative loss of about half a line of $\log$ MAR acuity when the non-dominant eye was corrected for $-1.00 \mathrm{D}$.

\section{Acknowledgments}

Steffen Østenstad, MSc, of IFocus Øyeklinikk AS assisted with diagnostic testing and data collection/checking. This work was supported by as an investigator-initiated study grant funded by Alcon (IIT\# 61478839).

\section{Disclosure}

Drs. Gundersen and Potvin are consultants to Alcon. The authors report no other conflicts of interest in this work.

\section{References}

1. Tarib I, Kasier I, Herbers C, et al. Comparison of visual outcomes and patient satisfaction after bilateral implantation of an EDOF IOL and a mix-and-match approach. J Refract Surg. 2019;35(7):408-416. PMID: 31298720. doi:10.3928/1081597X-20190417-02 
2. Sandoval HP, Potvin R, Solomon KD. Visual acuity, defocus curve, reading speed and patient satisfaction with a combined extended depth of focus intraocular lens and multifocal intraocular lens modality. Clin Ophthalmol. 2020;14:2667-2677. PMID: 32982159; PMCID: PMC7501984. doi:10.2147/OPTH.S276120

3. Pedrotti E, Carones F, Talli P, et al. Comparative analysis of objective and subjective outcomes of two different intraocular lenses: trifocal and extended range of vision. BMJ Open Ophthalmol. 2020;5: e000497. doi:10.1136/bmjophth-2020-000497

4. Hovanesian JA, Jones M, Allen Q. The PanOptix trifocal IOL vs the ReSTOR 2.5 active focus and ReSTOR 3.0-add multifocal lenses: a study of patient satisfaction, visual disturbances, and uncorrected visual performance. Clin Ophthalmol. 2021;15:983-990. PMID: 33692612; PMCID: PMC7939508. doi:10.2147/OPTH.S285628

5. Rodov L, Reitblat O, Levy A, Assia EI, Kleinmann G. Visual outcomes and patient satisfaction for trifocal, extended depth of focus and monofocal intraocular lenses. $J$ Refract Surg. 2019;35 (7):434-440. PMID: 31298723. doi:10.3928/1081597X-20190618-01

6. Cochener B, Boutillier G, Lamard M, Auberger-Zagnoli C. A comparative evaluation of a new generation of diffractive trifocal and extended depth of focus intraocular lenses. J Refract Surg. 2018;34 (8):507-514. PMID: 30089179. doi:10.3928/1081597X-20180530-02

7. Monaco G, Gari M, Di Censo F, Poscia A, Ruggi G, Scialdone A. Visual performance after bilateral implantation of 2 new presbyopia-correcting intraocular lenses: trifocal versus extended range of vision. J Cataract Refract Surg. 2017;43(6):737-747. PMID: 28732606. doi:10.1016/j.jcrs.2017.03.037

8. Singh BDO, Sharma SMD, Dadia FRCS, Suchit MS, Bharti NDNB, Bharti SMS. Comparative evaluation of visual outcomes after bilateral implantation of a diffractive trifocal intraocular lens and an extended depth of focus intraocular lens. Eye Contact Lens. 2020;46(5):314-318. doi:10.1097/ICL.0000000000000637

9. Sandoval HP, Lane S, Slade S, Potvin R, Donnenfeld ED, Solomon KD. Extended depth-of-focus toric intraocular lens targeted for binocular emmetropia or slight myopia in the nondominant eye: visual and refractive clinical outcomes. J Cataract Refract Surg. 2019;45 (10):1398-1403. PMID: 31444080. doi:10.1016/j.jcrs.2019.05.019

10. Hooshmand J, Allen P, Huynh T, et al. Small aperture IC-8 intraocular lens in cataract patients: achieving extended depth of focus through small aperture optics. Eye. 2019;33(7):1096-1103. PMID: 30792521; PMCID: PMC6707186. doi:10.1038/s41433-019-0363-9
11. Abdelrazek Hafez T, Helaly HA. Spectacle independence and patient satisfaction with pseudophakic mini-monovision using aberration-free intraocular lens. Clin Ophthalmol. 2019;13: 2111-2117. PMID: 31802840; PMCID: PMC6827509. doi:10.21 47/OPTH.S215229

12. Bellucci R, Cargnoni M, Bellucci C. Clinical and aberrometric evaluation of a new extended depth-of-focus intraocular lens based on spherical aberration. J Cataract Refract Surg. 2019;45(7):919-926. PMID: 31133419. doi:10.1016/j.jcrs.2019. 02.023

13. Vega F, Alba-Bueno F, Millán MS, Varón C, Gil MA, Buil JA. Halo and through-focus performance of four diffractive multifocal intraocular lenses. Invest Ophthalmol Vis Sci. 2015;56(6):3967-3975. Erratum in: Invest Ophthalmol Vis Sci. 2017 Sep 1;58(11):4694. PMID: 26098463. doi:10.1167/iovs.15-16600

14. US Food and Drug Administration. AcrySof ${ }^{\mathrm{TM}} \mathrm{IQ}$ vivity ${ }^{\mathrm{TM}}$ extended vision Intraocular Lens (IOL): summary of safety and effectiveness data. Available from: https://www.accessdata.fda.gov/cdrh_docs/pdf/ P930014S126B.pdf. Accessed March 7, 2021.

15. Cochener B. Influence of the level of monovision on visual outcome with an extended range of vision intraocular lens. Clin Ophthalmol. 2018;12:2305-2312. PMID: 30518994; PMCID: PMC6239114. doi:10.2147/OPTH.S184712

16. Barrett GD. Monovision with monofocal IOLs. In: Chang DF, editor. Mastering Refractive IOLs. The Art and Science. New Jersey: Slack; 2008:450-453.

17. Schojai M, Schultz T, Jerke C, Böcker J, Dick HB. Visual performance comparison of 2 extended depth-of-focus intraocular lenses. $J$ Cataract Refract Surg. 2020;46(3):388-393. PMID: 32142039. doi:10.1097/j.jcrs.0000000000000068

18. Tan J, Qin Y, Wang C, Yuan S, Ye J. Visual quality and performance following bilateral implantation of TECNIS symfony intraocular lenses with or without micro-monovision. Clin Ophthalmol. 2019;13:1071-1077. PMID: 31388295; PMCID: PMC6607220. doi:10.2147/OPTH.S202380

19. Turnbull AMJ, Hill WE, Barrett GD. Accuracy of intraocular lens power calculation methods when targeting low myopia in monovision. J Cataract Refract Surg. 2020;46(6):862-866. PMID: 32205728. doi:10.1097/j.jcrs.0000000000000187
Clinical Ophthalmology

\section{Publish your work in this journal}

Clinical Ophthalmology is an international, peer-reviewed journal covering all subspecialties within ophthalmology. Key topics include: Optometry; Visual science; Pharmacology and drug therapy in eye diseases; Basic Sciences; Primary and Secondary eye care; Patient Safety and Quality of Care Improvements. This journal is indexed on PubMed
Central and CAS, and is the official journal of The Society of Clinical Ophthalmology (SCO). The manuscript management system is completely online and includes a very quick and fair peer-review system, which is all easy to use. Visit http://www.dovepress.com/ testimonials.php to read real quotes from published authors. 